\title{
Construction of ceRNA Coexpression Network and Screening of Molecular Targets in Colorectal Cancer
}

\author{
Zhao Hui, ${ }^{1}$ Wang Zhanwei, ${ }^{2}$ Yang $\mathrm{Xi}^{3}{ }^{3}$ Liu Jin, ${ }^{4}$ Zhuang Jing, ${ }^{5}$ and Han Shuwen $\mathbb{D}^{3}$ \\ ${ }^{1}$ Department of Gastroenterology, Huzhou Central Hospital, Affiliated Central Hospital Huzhou University, 198 Hongqi Road, \\ Huzhou, Zhejiang, China 313000 \\ ${ }^{2}$ Department of Galactophore, Huzhou Central Hospital, Affiliated Central Hospital Huzhou University, 198 Hongqi Road, Huzhou, \\ Zhejiang, China 313000 \\ ${ }^{3}$ Department of Oncology, Huzhou Central Hospital, Affiliated Central Hospital Huzhou University, No. 198 Hongqi Road, Huzhou, \\ Zhejiang Province, China 313000 \\ ${ }^{4}$ Department of Pathology, Huzhou Central Hospital, Affiliated Central Hospital Huzhou University, No. 198 Hongqi Road, Huzhou, \\ Zhejiang Province, China 313000 \\ ${ }^{5}$ Graduate School of Nursing, Huzhou University, No. 1 Bachelor Road, Huzhou, Zhejiang Province 313000, China
}

Correspondence should be addressed to Han Shuwen; shuwenhan985@163.com

Received 17 January 2020; Accepted 2 April 2020; Published 21 April 2020

Academic Editor: Silvia Angeletti

Copyright ( 92020 Zhao Hui et al. This is an open access article distributed under the Creative Commons Attribution License, which permits unrestricted use, distribution, and reproduction in any medium, provided the original work is properly cited.

\begin{abstract}
Objective. To screen some RNAs that correlated with colorectal cancer (CRC). Methods. Differentially expressed miRNAs, lncRNAs, and mRNAs between cancer tissues and normal tissues in CRC were identified using data from the Gene Expression Omnibus (GEO) database. The Kyoto Encyclopedia of Genes and Genomes (KEGG) pathway and protein-protein interactions (PPIs) were performed to do the functional enrichment analysis. And a lncRNA-miRNA-mRNA network was constructed which correlated with CRC. RNAs in this network were subjected to analyze the relationship with the patient prognosis. Results. A total of 688, 241, and 103 differentially expressed genes (diff-mRNA), diff-lncRNA, and diff-miRNA were obtained between cancer tissues and normal tissues. A total of 315 edges were obtained in the ceRNA network. lncRNA RP11-108K3.2 and mRNA ONECUT2 correlated with prognosis. Conclusion. The identified RNAs and constructed ceRNA network could provide great sources for the researches of therapy of the CRC. And the lncRNA RP11-108K3.2 and mRNA ONECUT2 may serve as a novel prognostic predictor of CRC.
\end{abstract}

\section{Introduction}

Colorectal cancer (CRC) is a common malignant tumor in the gastrointestinal tract [1]. The initial syndrome of CRC is not conspicuous; but with the development of the tumor, the patient will show changes in bowel habits, blood in the stool, diarrhea, alternating diarrhea and constipation, local abdominal pain, and other symptoms. Patients with advanced symptoms often show syndromes such as anemia and weakness [2]. CRC is the third most common malignant tumor around the world, and its mortality rate is extremely high [3]. Considering the great threat to human health of CRC, a series of new diagnostic and therapeutic methods have emerged. CRC is a complex disease involving the expression and structure of genes [4]. More and more studies have shown that miRNA can play a crucial aspect in cancer progression by regulating its related targets, including mRNA and lncRNA [5].

lncRNA is a noncoding RNA with a length of more than 200 nucleotides, which acts a pivotal part in abounding actions such as dose compensation effect, epigenetic regulation, cell differentiation regulation, and cell cycle regulation $[6,7]$. It has been reported that upregulation of $\operatorname{lncRNA}$ FOXD3-AS1 suggests a lower survival rate in CRC patients. Experimental results showed that FOXD3-AS1 was overexpressed in CRC tissues and cells. Downregulation of FOXD3-AS1 expression in vitro can promote cell proliferation, invasion, and migration and promote apoptosis [8]. 
lncRNA-ATB and lncRNA-CCAT have strong accuracy in distinguishing CRC patients from healthy individuals [9]. miRNAs are endogenous noncoding RNAs with managerial actions, with a length of approximately 22 nucleotides that are involving posttranscriptional gene expression regulation in animals and plants [10]. miRNA-149 can be used as a target miRNA for identifying single bases in the serum of healthy and cancer patients. This method is direct and sensitive and can be used as an early diagnostic tool for colorectal cancer [11]. The expression of miR-139-5p was downregulated in the CRC cell line compared to the ordinary human colonic mucosal epithelial cell line NCM460. The study subsequently also demonstrated that overexpression of miR139-5p in colon tumor cell lines significantly inhibited proliferation of cells in vivo and in vitro. The final study found that miR-139-5p regulates chronic inflammation by inhibiting $\mathrm{NF}-\kappa \mathrm{B}$ activity, thereby inhibiting cell proliferation and invasion in CRC [12].

A series of studies have shown that miRNAs can silence gene by binding to mRNA, and IncRNA can regulate gene expression level relying on competitively binding miRNAs $[13,14]$. At this study, a IncRNA-miRNA-mRNA coexpression network was constructed using two GEO datasets to screen RNAs that may be associated with CRC and supply a novel method for the diagnosis and therapy of CRC (Figure 1).

\section{Materials and Methods}

2.1. Data Collection. The lncRNA/mRNA profile data GSE126092 was downloaded from Gene Expression Omnibus (GEO) database (https://www.ncbi.nlm.nih.gov/gds/?term=). And the extracted data were produced by the platform of Agilent-074348 Human lncRNA v6 $4 \times 180$ K (GPL21047, Probe Name Version). This dataset contained the data of lncRNA/mRNA expression profiles in 10 colorectal cancer (CRC) tissues and their corresponding normal-appearing tissues (NATs). The miRNA profile data GSE126093 was also extracted from GEO database. The data were produced by the platform of Exiqon miRCURY LNA microRNA array, 7th generation (GPL18058, miRBase v18, condensed Probe_ ID version). This dataset contained ten cases of CRC tissues and their corresponding NATs.

2.2. Data Preprocessing and Screening of Differentially Expressed $m R N A$, IncRNA, and miRNA (diff-mRNA, diff$\ln c R N A$, and diff-miRNA). Limma package was used to preprocess the downloaded raw data and screen the differentially expressed mRNA, lncRNA, and miRNA. The preprocess process included background correction, normalization, and concentration prediction. The matrix data was combined with the chip platform annotation file to map the probe to the symbol. For multiple probes corresponding to an equal symbol, the final expression was decided by the mean of probes. Differential expression analysis of tumor vs. control was performed on the samples using the classical Bayesian test and corrected with Benjamini/Hochberg. $p$ value $<0.05$ and $|\log 2 \mathrm{FC}|>2$ were taken as the cut-off for the screening of diff-mRNA, diff-lncRNA, and diff-miRNA.

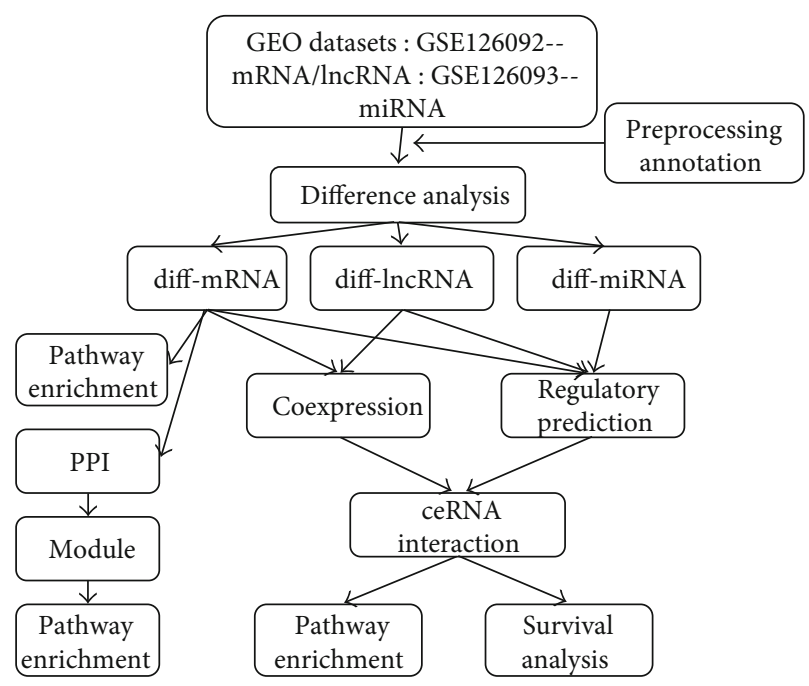

FIgURE 1: Diagram of bioinformatics analysis.

The heat maps of diff-mRNA, diff-lncRNA, and diff-miRNA were performed using heat map (version: 1.0.10, https://cran.r-project.org/web/packages/pheatmap/ index.html).

2.3. Analysis of Protein-Protein Interactions (PPIs) in diff$m R N A$. The PPIs of diff-mRNA analysis were performed using STRING (version: 10.0, https://www.string-db.org/) database with the score $=0.7$, which was visualized by Cytoscape (version: 3.2.0, http://www.cytoscape.org/). Furthermore, based on MCODE (version 1.4.2, http://apps.cytoscape.org/ apps/MCODE) with the score $\geq 10$, the network modules were obtained and evaluated from the original PPI network.

2.4. Functional Enrichment Analysis. The Kyoto Encyclopedia of Genes and Genomes (KEGG) pathways for diff-mRNA, mRNAs in modules that were significantly clustered in PPIs, and mRNAs in ceRNA network were carried out, via the $\mathrm{R}$ package clusterProfiler (version 2.4.3, http://bioconductor .org/packages/3.2/bioc/html/clusterProfiler.html). In KEGG pathway enrichment analysis, $p$ value $<0.05$ and gene count $>2$ were chosen as the cut-off criteria.

2.5. Prediction of diff-miRNA-diff-mRNA and diff-miRNAdiff-lncRNA. The miRWalk 2.0 (http://zmf.umm.uniheidelberg.de/apps/zmf/mirwalk2/) was used to presume the target miRNAs of the diff-miRNA. The selected genes must have results in six of the eight databases (miRanda, miRWalk, miRDB, Pictar2, miRMap, RNA22, PITA, and TargetScan). miRanda (version: 3.3a, https://omictools.com/miranda-tool) was used to analyze potential diff-miRNA-diff-lncRNA binding sites with the score $>150$ and energy $<-20$.

2.6. Coexpression Analysis of diff-lncRNA and diff-mRNA. The Pearson correlation coefficient (PCC) was calculated between lncRNA and mRNA. And the PPC $>0.95$ and $p$ value $<0.05$ were considered to be meaningful correlation. Cytoscape was used to illustrate the coexpression network. 


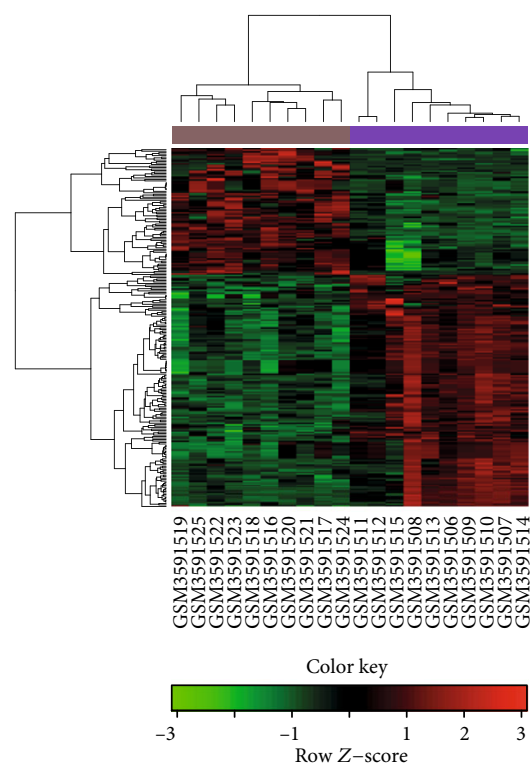

(a)

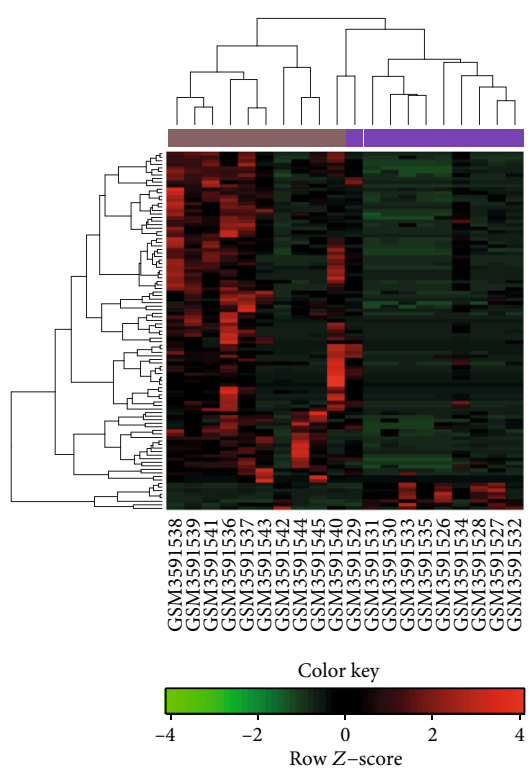

(b)

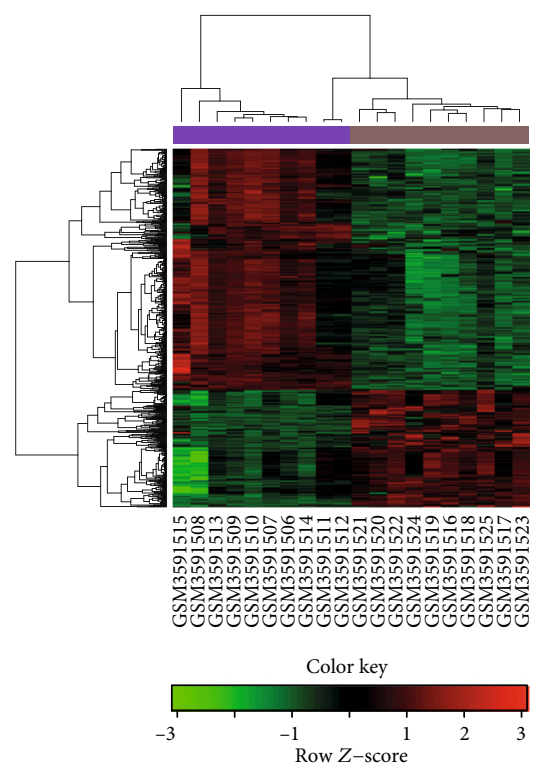

(c)

FIGURE 2: The heat maps of the differentially expressed lncRNAs, miRNAs, and mRNAs in CRC. The relative expression of the differentially expressed lncRNAs (a), miRNAs (b), and mRNAs (c), respectively, between the cancer tissues and normal tissues. Green indicates downregulated RNAs, and red indicates upregulated RNAs.

2.7. Survival Analysis. The colon cancer tumor samples from which mRNA, IncRNA, miRNA, and survival prognosis messages were extracted from the TCGA database, and the expression values and survival prognosis information of all node elements in the IncRNA-miRNA-mRNA network were extracted from the TCGA colon cancer samples. K-M survival curve was performed using Survival (version: 2.42-6, https:// cran.r-project.org/web/packages/survival/index.html). $p$ value $<0.05$ was considered as the significant threshold.

\section{Results}

3.1. Clustering of Differentially Expressed RNAs in CRC Tissues. A total of 688, 241, and 103 differentially expressed mRNAs (diff-mRNAs), diff-lncRNAs, and diff-miRNAs were obtained, respectively, including 266 upregulated diff-mRNA, 462 downregulated diff-mRNA, 85 upregulated diff-lncRNA, 156 downregulated diff-lncRNA, 103 upregulated diff-miRNA, and 95 downregulated diff-miRNA. As shown in Figure 2, the two groups of samples could be separated significantly.

The top 10 expression of diff-mRNA was MMP7, LEMD1, CLDN1, SLCO1B3, REG1A, COL10A1, ETV4, COL11A1, APOBEC1, and LGR5. The top 10 expression of the following diff-IncRNA was CCAT1, LOC401585, AC123023.1, UCA1, EVADR, RP11-132A1.4, CTD-2008A1.3, SNAR-H, SNARC1, and SNAR-E. The top 10 expression of diff-miRNA was hsa-miR-31-5p, hsa-miR-224-5p, hsa-miR-1244, hsa-miR188-5p, hsa-miR-764, hsa-miR-301b, hsa-miR-19b-1-5p, hsa-miR-3648, hsa-miR-452-3p, and hsa-miR-3157-3p. The details were shown in Supplementary tables 1, 2, and 3.

3.2. Screening the Cell Pathways with regard to Differentially Expressed mRNA in CRC. KEGG pathway analysis was per- formed to make a deep understanding between the diffmRNA and cell pathways. The result indicated that all 266 upregulated diff-mRNAs were enriched in 10 pathways, including cell cycle, oocyte meiosis, progesterone-mediated oocyte, and cytokine-cytokine receptor interaction. And the 462 downregulated diff-mRNAs were enriched in 21 pathways, including hypertrophic cardiomyopathy, dilated cardiomyopathy, cGMP-PKG signaling pathway, and cAMP signaling pathway (Table 1).

3.3. PPI Network with regard to Differentially Expressed $m R N A s$ in CRC. PPI network was constructed, which integrates large amount of known and predicted interactions of proteins. As shown in Figure 3, 354 nodes and 1,500 edges were included in the network. Module analysis was performed using MCODE, and two modules were obtained (module A and module B). Module A (score $=32.778$ ) was constructed with 37 nodes and 590 edges, and module B (score $=15)$ was constructed with 15 nodes and 105 edges (Figures 4(a) and 4(b)). KEGG analysis was also performed. The result indicated that 37 diff-mRNAs in module A were enriched in 4 pathways, and 15 diff-mRNAs in module B were enriched in 8 pathways (Table 2 ).

\section{4. miRNA-lncRNA/mRNA Prediction and $\ln c R N A-m R N A$} Coexpression. The top 10 upregulated and all 8 downregulated diff-miRNAs were predicted using miRWalk 2.0 database. The edges were screened which the target genes were diff-mRNA. And a total of 308 edges of diff-miRNA-diffmRNAs were obtained finally, including 15 diff-miRNAs and 177 diff-mRNAs. Thirty-nine edges of lncRNA-miRNA were obtained including 10 diff-miRNAs and 33 difflncRNAs. Furthermore, 143 target genes regulated by 10 
TABLE 1: KEGG enrichment pathway.

\begin{tabular}{|c|c|c|c|}
\hline & KEGG pathway & Count & $p$ value \\
\hline \multirow{10}{*}{ Upregulated } & hsa04110: cell cycle & 15 & $1.09 E-09$ \\
\hline & hsa04114: oocyte meiosis & 10 & $1.98 E-05$ \\
\hline & hsa04914: progesterone-mediated oocyte maturation & 9 & $2.35 E-05$ \\
\hline & hsa04060: cytokine-cytokine receptor interaction & 14 & $2.56 E-05$ \\
\hline & hsa05322: systemic lupus erythematosus & 8 & $2.42 E-03$ \\
\hline & hsa05323: rheumatoid arthritis & 6 & $7.18 E-03$ \\
\hline & hsa05034: alcoholism & 8 & $1.09 E-02$ \\
\hline & hsa04062: chemokine signaling pathway & 8 & $1.41 E-02$ \\
\hline & hsa05132: Salmonella infection & 5 & $2.74 E-02$ \\
\hline & hsa03460: Fanconi anemia pathway & 4 & $3.64 E-02$ \\
\hline \multirow{21}{*}{ Downregulated } & hsa05410: hypertrophic cardiomyopathy (HCM) & 9 & $7.97 E-04$ \\
\hline & hsa05414: dilated cardiomyopathy & 9 & $1.30 E-03$ \\
\hline & hsa04022: cGMP-PKG signaling pathway & 12 & $2.32 E-03$ \\
\hline & hsa04024: cAMP signaling pathway & 13 & $4.68 E-03$ \\
\hline & hsa04080: neuroactive ligand-receptor interaction & 16 & $4.74 E-03$ \\
\hline & hsa04020: calcium signaling pathway & 12 & $6.04 E-03$ \\
\hline & hsa04724: glutamatergic synapse & 9 & $8.61 E-03$ \\
\hline & hsa04261: adrenergic signaling in cardiomyocytes & 10 & $8.69 E-03$ \\
\hline & hsa04713: circadian entrainment & 8 & $1.06 E-02$ \\
\hline & hsa00980: metabolism of xenobiotics by cytochrome $\mathrm{P} 450$ & 7 & $1.13 E-02$ \\
\hline & hsa04911: insulin secretion & 7 & $2.13 E-02$ \\
\hline & hsa00071: fatty acid degradation & 5 & $2.18 E-02$ \\
\hline & hsa04725: cholinergic synapse & 8 & $2.33 E-02$ \\
\hline & hsa04726: serotonergic synapse & 8 & $2.33 E-02$ \\
\hline & hsa00830: retinol metabolism & 6 & $2.34 E-02$ \\
\hline & hsa04978: mineral absorption & 5 & $2.54 E-02$ \\
\hline & hsa05412: arrhythmogenic right ventricular cardiomyopathy (ARVC) & 6 & $2.79 E-02$ \\
\hline & hsa03320: PPAR signaling pathway & 6 & $2.79 E-02$ \\
\hline & hsa04920: adipocytokine signaling pathway & 6 & $3.29 E-02$ \\
\hline & hsa04921: oxytocin signaling pathway & 9 & $3.82 E-02$ \\
\hline & hsa04728: dopaminergic synapse & 8 & $4.54 E-02$ \\
\hline
\end{tabular}

miRNAs in the lncRNA-miRNA regulatory network were screened and coexpressed with 33 lncRNAs in the lncRNAmiRNA regulatory network. Based on the screening threshold, 77 edges of positive correlation lncRNA-mRNA were obtained.

3.5. IncRNA-miRNA-mRNA Network. According to the whole relationship of edges above, the lncRNA-miRNA-mRNA edges were further collated. A total of 315 edges were obtained, of which 77 lncRNA-mRNA positive correlation coexpression edges, 199 miRNA-mRNA regulatory edges, and 39 lncRNAmiRNA regulatory edges. And all the edges contained 186 nodes, 7 up-miRNAs, 3 down-miRNAs, 34 up-mRNAs, 109 down-mRNAs, 16 up-lncRNAs, and 17 down-lncRNAs.

lncRNA-miRNA-mRNA network is shown in Figure 5, and the nodes in the network are shown in Supplementary Table 4. The diff-mRNAs in IncRNA-miRNA-mRNA network were analyzed using KEGG pathway enrichment analysis, of which 15 pathways (Table 3 ).
3.6. Differentially Expressed RNAs with regard to the Prognosis of CRC. All the nodes in Figure 6 were used to do the survival analysis, of which a lncRNA RP11-108K3.2 and one mRNA ONECUT2 correlated with prognosis.

\section{Discussion}

In this study, to screen for RNAs and pathways associated with CRC, a lncRNA-miRNA-mRNA expression network was constructed using two datasets downloaded from the GEO database. A total of 688 diff-mRNAs, 241 diff-lncRNAs, and 103 diff-miRNAs were identified at the present study. A functional enrichment analysis of these 688 diff-mRNAs revealed that they were mainly enriched in 21 pathways. A PPI network analysis was also performed on these diffmRNAs. Finally, a ceRNA network was constructed and a survival analysis was performed on the nodes to obtain two prognostic-related RNAs. The expression levels of RP11108K3.2 and ONECUT2 were both upregulated in CRC, 


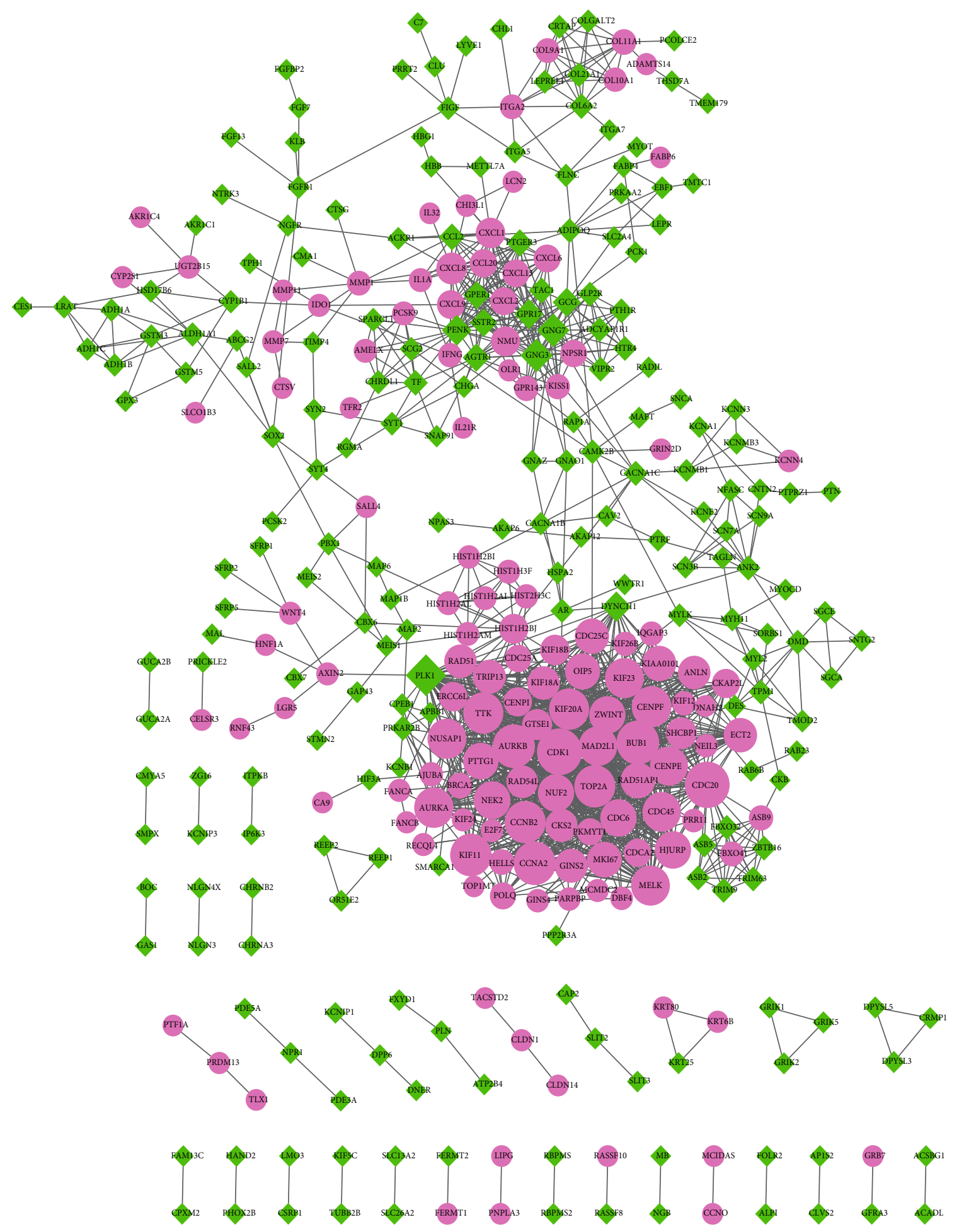

FIGURE 3: The protein-protein interaction network of differentially expressed mRNAs. The purple nodes represented the upregulated mRNAs, and the green nodes represented the downregulated mRNAs. The higher the degree value is, the larger the nodes.

and low levels were associated with better prognosis, suggesting that they may play a positive role in CRC.

lncRNA can participate in the initiation and development of many diseases by directly regulating proteins or indirectly regulating the target genes of related miRNA. The result of ceRNA showed that RP11-108K3.2 was regulated by hsa-miR-224-5p. Study has shown that the great expression level of RP11-108K3.2 correlated with low overall survival of CRC, and this result was consistent with our study [15]. In a study on lung adenocarcinoma, the researchers study 346 differentially expressed lncRNAs, of which RP11108K3.2 is highly expressed in lung cancer tissues [16]. 


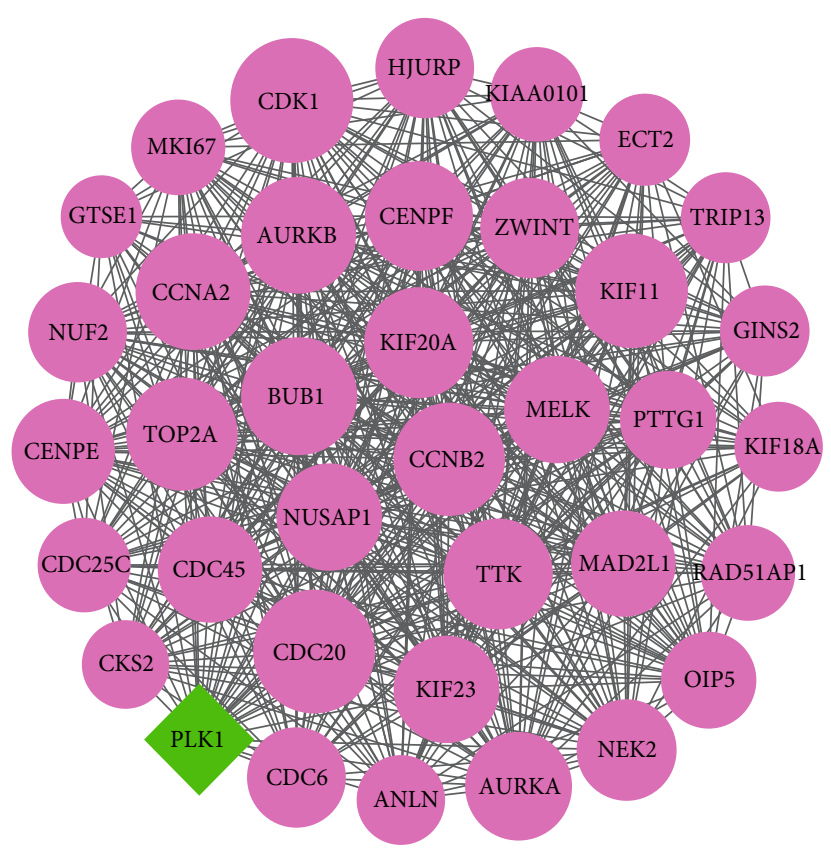

(a)

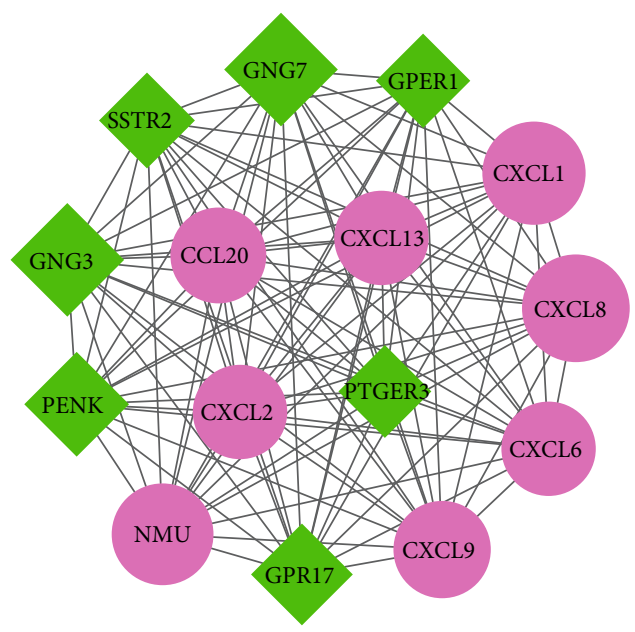

(b)

FiguRE 4: The protein-protein interaction network of differentially expressed mRNAs in module A (a) and module B (b). The purple nodes represented the upregulated mRNAs, and the green nodes represented the downregulated mRNAs. The higher the degree value is, the larger the nodes.

TABLE 2: KEGG enrichment pathway of modules.

\begin{tabular}{|c|c|c|c|}
\hline & KEGG pathway & Count & $p$ value \\
\hline \multirow{4}{*}{ Module-A } & hsa04110: cell cycle & 12 & $5.33 E-17$ \\
\hline & hsa04114: oocyte meiosis & 9 & $2.09 E-11$ \\
\hline & hsa04914: progesterone-mediated oocyte maturation & 7 & $1.57 E-08$ \\
\hline & hsa04115: p53 signaling pathway & 3 & $9.04 E-03$ \\
\hline \multirow{8}{*}{ Module-B } & hsa04062: chemokine signaling pathway & 9 & $3.79 E-11$ \\
\hline & hsa04060: cytokine-cytokine receptor interaction & 7 & $7.28 E-07$ \\
\hline & hsa05134: legionellosis & 3 & $3.18 E-03$ \\
\hline & hsa04621: NOD-like receptor signaling pathway & 3 & $3.42 E-03$ \\
\hline & hsa05132: Salmonella infection & 3 & $7.37 E-03$ \\
\hline & hsa05323: rheumatoid arthritis & 3 & $8.26 E-03$ \\
\hline & hsa04668: TNF signaling pathway & 3 & $1.20 E-02$ \\
\hline & hsa05200: pathways in cancer & 4 & $2.17 E-02$ \\
\hline
\end{tabular}

Furthermore, hsa-miR-224-5p is upregulated both in adenoma and in cancer tissues [17]. A study has been revealed that hsa-miR-224-5p could suppress the cell growth of two oral squamous cell carcinoma cell lines (SCC4, SCC15). However, when hsa-miR-224-5p and pcDNA3.1-CT-GFPFTH1P3 are cotransfected, the growth inhibition of hsamiR-224-5p on oral squamous cell carcinoma will be reversed [18]. In this study, the expressions of RP11$108 \mathrm{~K} 3.2$ and hsa-miR-224-5p were both upregulated in CRC samples. This result indicates that lncRNA RP11$108 \mathrm{~K} 3.2$ and hsa-miR-224-5p may play a same role as hsamiR-224-5p and FTH1P3.

ONECUT2 is a member of the ONECUT2 transcription factor family that interacted with hsa-miR-139-5p, hsa-
miR-188-5p, hsa-miR-19b-1-5p, hsa-miR-31-5p, and hsamiR-497-5p in a ceRNA network in this study [19]. It has been reported that ONECUT2 acts a critical position on CRC gene network and is significantly associated with the development of cancer. Interference with endogenous ONECUT2 expression inhibited the CRC cell line SW620 cell migration [20]. A series of experiments have shown that hsa-miR-139-5p acts as a crucial part in colon tumor. Compared with normal tissues, the hsa-miR-139-5p expression level is significantly declined in colon tumor, and its expression level is significantly correlated with tumor stage. Subsequently, experiments have shown that the direct object of hsa-miR-139-5p is BCL2, and the expression of BCL2 is negatively correlated with the expression of hsa-miR-139-5p. 


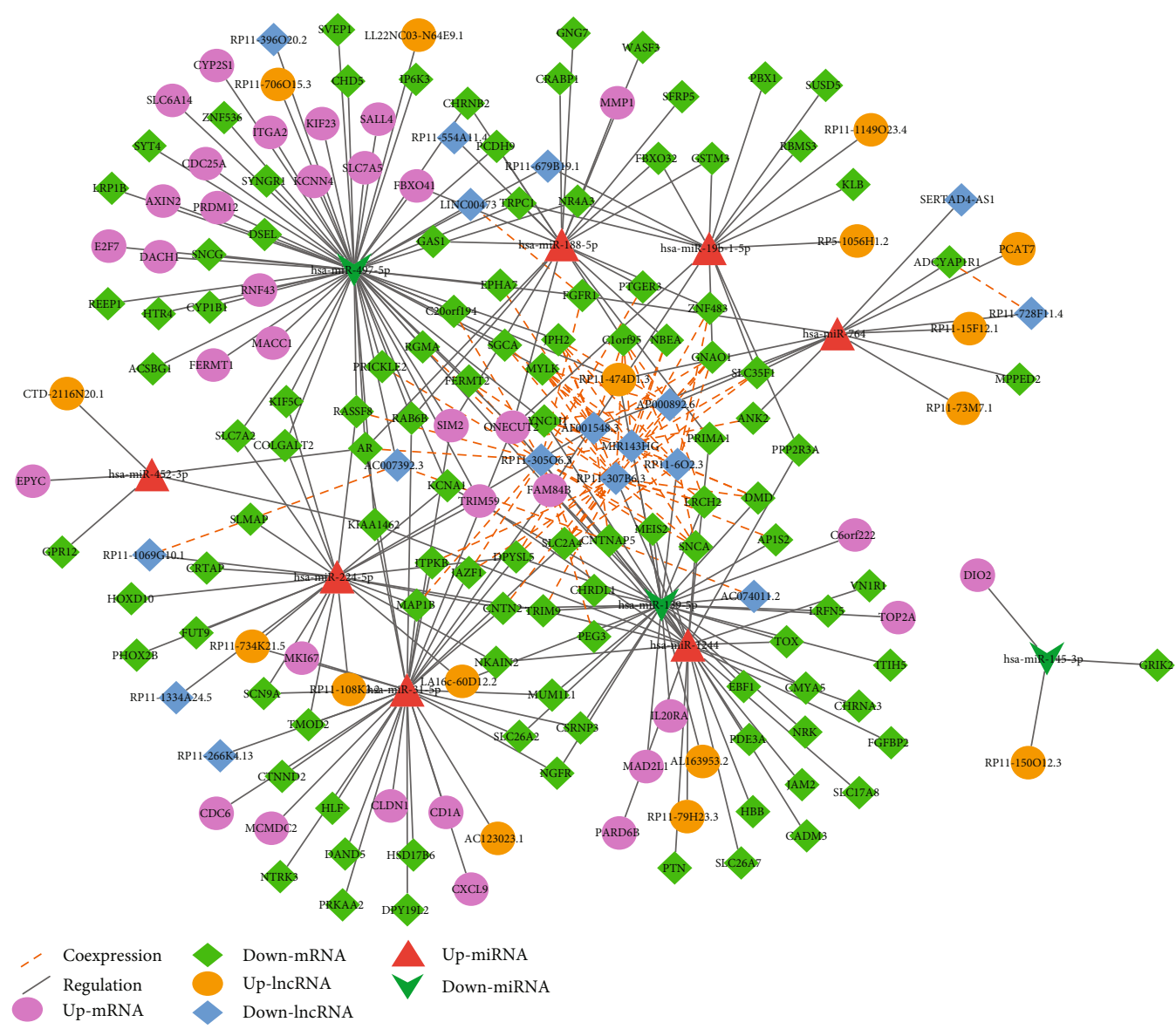

FIgURE 5: ceRNA network in CRC. The purple ellipse and the green diamond represented the up- and downregulated mRNAs, respectively. The orange ellipse and the blue diamond represented the up- and downregulated lncRNAs, respectively. The red triangle and the dark green arrow represented the up- and downregulated miRNAs, respectively. The red dotted line represented the coexpression relationships, and the black line represented the regulation relationships.

TABLE 3: KEGG enrichment pathway of diff-mRNAs in ceRNA network.

\begin{tabular}{lcc}
\hline KEGG pathway & $p$ value & Count \\
\hline $\begin{array}{l}\text { hsa04724: glutamatergic synapse } \\
\text { hsa05410: hypertrophic cardiomyopathy }\end{array}$ & $3.40 E-03$ & 5 \\
$\begin{array}{l}\text { (HCM) } \\
\text { hsa04725: cholinergic synapse }\end{array}$ & $1.85 E-03$ & 4 \\
hsa04530: tight junction & $1.76 E-02$ & 4 \\
hsa04726: serotonergic synapse & $1.92 E-02$ & 4 \\
hsa05230: central carbon metabolism in cancer & $2.01 E-02$ & 3 \\
hsa04360: axon guidance & $2.24 E-02$ & 5 \\
hsa04920: adipocytokine signaling pathway & $2.35 E-02$ & 3 \\
hsa05412: arrhythmogenic right & $2.62 E-02$ & 3 \\
ventricular cardiomyopathy (ARVC) & & \\
hsa04728: dopaminergic synapse & $2.93 E-02$ & 4 \\
hsa00980: metabolism of xenobiotics by & $3.01 E-02$ & 3 \\
cytochrome P450 & $3.94 E-02$ & 4 \\
hsa04514: cell adhesion molecules (CAMs) & $4.74 E-02$ & 3 \\
hsa05032: morphine addiction & $4.74 E-02$ & 3 \\
hsa05414: dilated cardiomyopathy (DCM) & $4.93 E-02$ & 2 \\
hsa05033: nicotine addiction &
\end{tabular}

The tumor metastasis and drug sensitivity of CRC could be diminished by hsa-miR-139-5p targeting the BCL2 pathway [21]. In the present study, hsa-miR-139-5p was interacted with ONECUT2, and its expression level was negatively connected with the expression level of the later one. These two RNAs may have a similar mechanism with hsa-miR-139-5p and BCL2. hsa-miR-31-5p as an infrequently expressed miRNA was associated with a stage when assessing CRC cases. The upregulated hsa-miR-31-5p makes a more advanced disease stage more likely than a lower disease stage [22]. At this research, the expression level of hsa-miR-31-5p was higher in CRC tissues than in control $(\log F C=9.153176)$. In addition to being significantly upregulated in CRC tissues, it has been reported to be significantly upregulated in uterine cervical cancer tissues [23].

In conclusion, at the present study, several CRC-related diff-miRNAs, diff-lncRNAs, and diff-mRNAs were obtained. A ceRNA network was also constructed to analyze the crosstalk among the identified diff-miRNAs, diff-lncRNAs, and diff-mRNAs. The lncRNA RP11-108 K3.2 and mRNA ONECUT2 in the network may play a crucial role in CRC, with their low expression levels being correlated with better prognosis. Although the mechanism of RP11-108K3.2 and ONECUT2 remains to be revealed, both of the two may be used as 


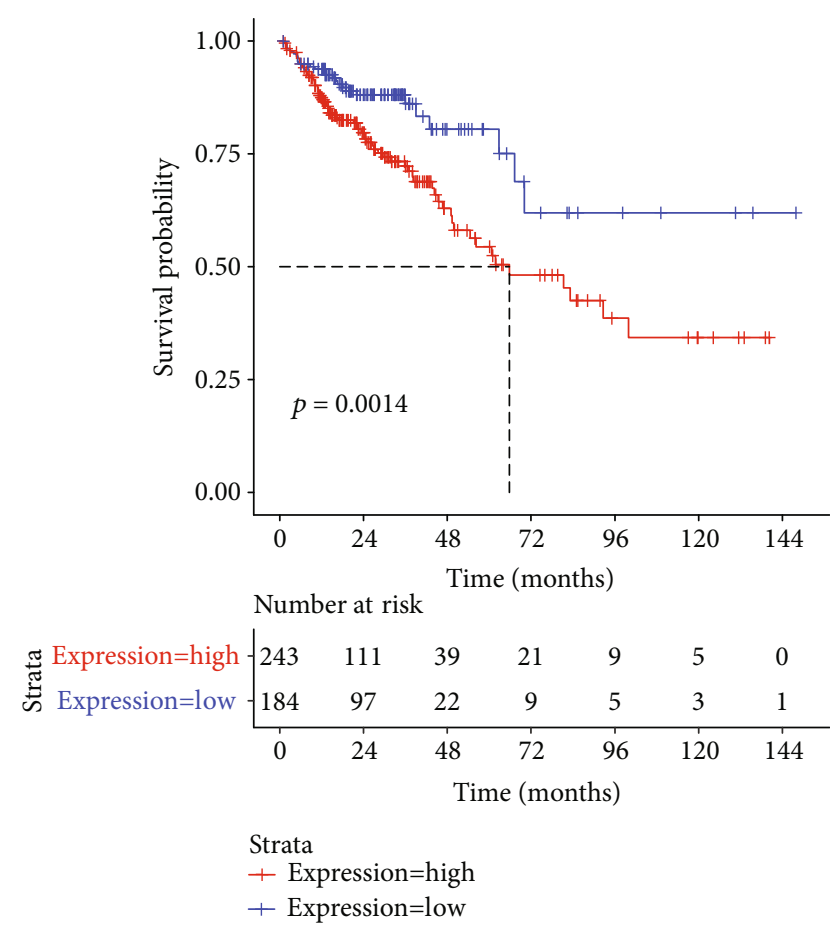

(a)

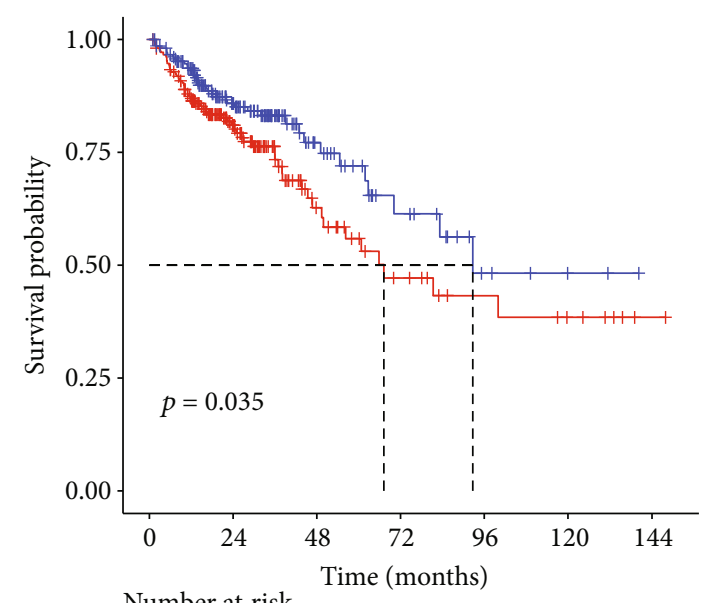

㞼 Expression=high Expression=low $\begin{array}{ccccccc}-215 & 94 & 29 & 15 & 9 & 6 & 1 \\ -212 & 114 & 32 & 15 & 5 & 2 & 0 \\ 0 & 24 & 48 & 72 & 96 & 120 & 144 \\ \text { Time (months) }\end{array}$

(b)

FIGURE 6: Survival curve with CRC containing differentially expressed RNAs. The mRNA, lncRNA, and miRNA expression levels and patient survival information from TCGA CRC data were used to plot the KM survival curve. The figure showed that the lncRNA RP11-108 K3.2 (a) and mRNA ONECUT2 (b) significantly correlated with prognosis of CRC.

novel clinical predictors of CRC. Further experimental studies are needed to reveal the mechanism of RP11-108K3.2 and ONECUT2 in CRC in the future.

\section{Data Availability}

Answer: Yes. Comment: The datasets generated during the current study are not publicly available but identified and anonymized information is potentially available on reasonable request.

\section{Additional Points}

Highlights. (1) A total of 688, 241, and 103 differentially expressed mRNA (diff-mRNA), diff-lncRNA, and diffmiRNA were obtained. (2) In the ceRNA network, a total of 315 edges were obtained. (3) lncRNA RP11-108K3.2 and mRNA ONECUT2 correlated with prognosis

\section{Conflicts of Interest}

The authors declare that no conflicts of interest exist.

\section{Authors' Contributions}

All authors participated in the conception and design of the study; Han Shuwen conceived the manuscript; Wang Zhanwei and Yang Xi processed the data; Zhao Hui, Liu Jin, and Zhuang Jing analyzed the data; Zhao Hui and Zhuang Jing wrote the paper; all authors read and approved the paper.

\section{Acknowledgments}

This work was supported by the Zhejiang Science and Technology Projects (No. Q20H160002).

\section{Supplementary Materials}

Supplementary table 1: the differentially expressed lncRNAs (diff-lncRNAs). Supplementary table 2: the differentially expressed miRNAs (diff-miRNAs). Supplementary table 3: the differentially expressed mRNAs (diff-mRNAs). Supplementary table 4 : the differentially expressed miRNAs (diffmiRNAs). (Supplementary Materials)

\section{References}

[1] S. Yan, Y. Cao, and A. Mao, "MicroRNAs in colorectal cancer: potential biomarkers and therapeutic targets," Frontiers in Bioscience, vol. 20, no. 7, pp. 1092-1103, 2015.

[2] J. J. Puente Gutiérrez, J. L. Domínguez Jiménez, M. A. Marín Moreno, and E. Bernal Blanco, "Valor de la indicación de la colonoscopia como predictor de diagnóstico de cáncer colorrectal. ¿Se puede diseñar un circuito rápido de diagnóstico?," Gastroenterología Y Hepatología, vol. 31, no. 7, pp. 413-420, 2008.

[3] B. Baran, N. Mert Ozupek, N. Yerli Tetik, E. Acar, O. Bekcioglu, and Y. Baskin, "Difference between left-sided and right-sided colorectal cancer: a focused review of Literature," Gastroenterology Research, vol. 11, no. 4, pp. 264-273, 2018. 
[4] X. Han, E. Clark, P. M. Shaw et al., "Transcriptional characteristics of genomic instabilities for colorectal cancer patients," Cancer Research, vol. 65, pp. 840-840, 2005.

[5] N. B. Hao, Y. F. He, X. Q. Li, K. Wang, and R. L. Wang, "The role of miRNA and lncRNA in gastric cancer," Oncotarget, vol. 8, no. 46, pp. 81572-81582, 2017.

[6] A. Renganathan, J. Kresoja-Rakic, N. Echeverry et al., "GAS5 long non-coding RNA in malignant pleural mesothelioma," Molecular Cancer, vol. 13, no. 1, p. 119, 2014.

[7] H. E. Johansson, A. V. Orjalo Jr., S. R. Coassin, J. L. Ruth, F. Stossi, and M. A. Mancini, "Abstract 1940: high-throughput imaging and high content analysis of disease relevant lncRNAs examined by RNA fluorescence in situ hybridization (RNA FISH)," Cancer Research, vol. 73, Supplement 8, pp. 19401940, 2013.

[8] Q. Wu, M. Shi, W. Meng, Y. Wang, P. Hui, and J. Ma, "Long noncoding RNA FOXD3-AS1 promotes colon adenocarcinoma progression and functions as a competing endogenous RNA to regulate SIRT1 by sponging miR-135a-5p," Journal of Cellular Physiology, vol. 234, no. 12, pp. 21889-21902, 2019.

[9] P. Abedini, A. Fattahi, S. Agah et al., "Expression analysis of circulating plasma long noncoding RNAs in colorectal cancer: the relevance of lncRNAs ATB and CCAT1 as potential clinical hallmarks," Journal of Cellular Physiology, vol. 234, no. 12, pp. 22028-22033, 2019.

[10] Y. Luan, J. Cui, J. Zhai, J. Li, L. Han, and J. Meng, "Highthroughput sequencing reveals differential expression of miRNAs in tomato inoculated with Phytophthora infestans," Planta, vol. 241, no. 6, pp. 1405-1416, 2015.

[11] H.-N. Chan, S.-L. Ho, D. He, and H. W. Li, "Direct and sensitive detection of circulating miRNA in human serum by ligasemediated amplification," Talanta, vol. 206, article 120217, 2020.

[12] M. Zhu, W. Zhang, J. Ma et al., "MicroRNA-139-5p regulates chronic inflammation by suppressing nuclear factor- $\kappa \mathrm{B}$ activity to inhibit cell proliferation and invasion in colorectal cancer," Experimental and Therapeutic Medicine, vol. 18, no. 5, pp. 4049-4057, 2019.

[13] X. Shi, Y. Xu, C. Zhang et al., "Subpathway-LNCE: identify dysfunctional subpathways competitively regulated by lncRNAs through integrating lncRNA-mRNA expression profile and pathway topologies," Oncotarget, vol. 7, no. 43, pp. 69857-69870, 2016.

[14] H. Liang, T. Yu, Y. Han et al., "LncRNA PTAR promotes EMT and invasion-metastasis in serous ovarian cancer by competitively binding miR-101-3p to regulate ZEB1 expression," Molecular Cancer, vol. 17, no. 1, p. 119, 2018.

[15] W. Zhou, B. Pan, and L. Liu, "Integrated bioinformatics analysis revealing independent prognostic long non-coding RNAs DNAH17-AS1 and RP11-400N13.2 and their potential oncogenic roles in colorectal cancer," Oncology Letters, vol. 18, no. 4, pp. 3705-3715, 2019.

[16] X. Shi, H. Tan, X. Le et al., "An expression signature model to predict lung adenocarcinoma-specific survival," Cancer Management and Research, vol. 10, pp. 3717-3732, 2018.

[17] H. Jin, wang, L. Chen et al., "Screening miRNAs for early diagnosis of colorectal cancer by small RNA deep sequencing and evaluation in a Chinese patient population," OncoTargets and Therapy, vol. 9, article 1159, 2016.

[18] C.-Z. Zhang, "Long non-coding RNA FTH1P3 facilitates oral squamous cell carcinoma progression by acting as a molecular sponge of miR-224-5p to modulate fizzled 5 expression," Gene, vol. 607, pp. 47-55, 2017.

[19] F. Wu, D. Sapkota, R. Li, and X. Mu, "Onecut 1 and Onecut 2 are potential regulators of mouse retinal development," Journal of Comparative Neurology, vol. 520, no. 5, pp. 952-969, 2012.

[20] Y. Sun, S. Shen, X. Liu et al., "MiR-429 inhibits cells growth and invasion and regulates EMT-related marker genes by targeting Onecut2 in colorectal carcinoma," Molecular and Cellular Biochemistry, vol. 390, no. 1-2, pp. 19-30, 2014.

[21] Q. Li, X. Liang, Y. Wang et al., "miR-139-5p inhibits the epithelial-mesenchymal transition and enhances the chemotherapeutic sensitivity of colorectal cancer cells by downregulating BCL2," Scientific Reports, vol. 6, no. 1, article 27157, 2016.

[22] M. L. Slattery, A. J. Pellatt, F. Y. Lee et al., "Infrequently expressed miRNAs influence survival after diagnosis with colorectal cancer," Oncotarget, vol. 8, no. 48, pp. 8384583859, 2017.

[23] V. B. Nair, V. G. Manasa, M. S. Sinto, K. Jayasree, F. V. James, and S. Kannan, "Differential expression of microRNAs in uterine cervical cancer and its implications in carcinogenesis; an integrative approach," International Journal of Gynecological Cancer, vol. 28, no. 3, pp. 553-562, 2018. 